\title{
Does the locus on chromosome 11 implicated in susceptibility to HLA-DR4 dependent type I diabetes mellitus also affect susceptibility to rheumatoid arthritis?
}

\author{
K D Pile, B P Wordsworth, J I Bell
}

\begin{abstract}
There is a polygenic component to rheumatoid arthritis (RA) in addition to the known association with HLA-DR4. It has previously been shown in another autoimmune disease (type I diabetes mellitus) that a gene on chromosome 11p can act with HLA-DR4 to enhance susceptibility (relative risk 5.6). It is therefore possible that this locus may also affect the development of RA. Genotytpe frequencies at this locus, defined by a dimorphic Fok 1 restriction site, were compared in 139 healthy controls and 213 patients with classical/definite $\mathbf{R A}$. In contrast with diabetes there was no increase in genotypes lacking the Fok 1 site, either in the rheumatoid group overall (125/211 compared with 86/139 controls) or in the DR4 positive rheumatoid group (76/140 compared with controls). These results indicate that the interaction between DR4 and a locus on chromosome $11 p$ is not common to all DR4 associated autoimmune diseases.
\end{abstract}

(Ann Rheum Dis 1992; 51: 1250-1251)

Rheumatoid arthritis (RA) is a complex disease with a contributory polygenic component. ${ }^{1}$ There are strong associations between RA and the HLA-DR4 antigen in most ethnic groups, ${ }^{2} 3$ particularly in subjects with severe disease. ${ }^{4}$ Furthermore, in this group of patients there is an increase in DR4 homozygotes, resulting particularly from an increase in DR4Dw4/ DR4Dw14 genotypes. ${ }^{45}$ Despite these observations it has been estimated that no more than one third of the total genetic contribution to RA arises from HLA linked genes. ${ }^{16}$ Observations in type I diabetes mellitus, another autoimmune disease with strong HLA associations, have implicated a second gene on chromosome 11 in susceptibility to this disease, in the region containing the insulin gene and insulin-like growth factor 2 . The effect of this locus is only manifest in HLA-DR4 positive subjects, however. ${ }^{7}$ DR4 positive subjects who are also homozygous for haplotypes lacking a Fok 1 restriction site in the DNA flanking the $3^{\prime}$ end of the insulin gene have a relative risk of $5 \cdot 1$ for the development of type I diabetes. We have therefore investigated the possibility that this locus may also influence susceptibility to RA, particularly in those subjects who carry the DR4 antigen.

Subjects, methods, and results

We studied 139 healthy controls and 211 patients with classical/definite rheumatoid factor positive RA, all of whom had progressive erosive arthritis requiring treatment with second line drugs, and 140 of whom were DR4 positive. HLA-DR genotypes were determined for all the subjects with RA by the amplification of DRB1 alleles from genomic DNA by the polymerase chain reaction and serial hybridisation to radiolabelled oligonucleotides as described previously. ${ }^{8}$

Genotypes for the insulin/insulin-like growth factor 2 region were defined using a dimorphic Fok 1 restriction enzyme. ${ }^{7}$ A 568 base pair fragment of DNA flanking the $3^{\prime}$ end of the insulin gene was amplified by the polymerase chain reaction using two oligonucleotide primers (a) 5'-CAGCCCAGCCTCCTCCCTCCACA-3' and (b) 5'-TGCAGAAGCGTGGCATTGTG GAAC $-3^{\prime}$. Ten microlitres of the final reaction mix was digested with Fok 1 in a total reaction volume of $30 \mu \mathrm{l}$ according to the manufacturer's instructions (New England Biolabs, Beverly, MA, USA). The products were separated by agarose gel electrophoresis and visualised after staining with ethidium bromide under ultraviolet light. Absence of the Fok 1 restriction site yielded a band of 568 base pairs that was cleaved to bands at 386 and 182 base pairs when the site was present.

The distribution of individual genotypes in
Molecular Immunology Group, Institute of Molecular Medicine, John Radcliffe Hospital, Oxford OX3 9DU, United Kingdom K D Pile B P Wordsworth J T Bell

Correspondence to: Dr Wordsworth.

Accepted for publication 24 June 1992

Relative risks with 95\% confidence intervals for Fok 1 genotypes at the insulin/insulin-like growth factor 2 locus in all patients with rheumatoid arthritis $(R A)$ and in $D R 4$ positive patients with $R A$

\begin{tabular}{|c|c|c|c|c|}
\hline \multirow[t]{2}{*}{ Subject group } & \multicolumn{2}{|c|}{ Genotypes } & \multirow[t]{2}{*}{ Relative risk } & \multirow[t]{2}{*}{$\chi^{2: *}$} \\
\hline & 2,2 & 1,2 or 1,1 & & \\
\hline $\begin{array}{l}\text { Controls }(n=139) \\
\text { All patients with RA }(n=211) \\
\text { Patients with RA positive for DR4 }(n=140)\end{array}$ & $\begin{array}{l}86 \\
125 \\
76\end{array}$ & $\begin{array}{l}53 \\
86 \\
64\end{array}$ & $\begin{array}{l}\overline{0.9}(0.5 \text { to } 1.6) \\
0.7(0.4 \text { to } 1 \cdot 2)\end{array}$ & $\begin{array}{l}\overline{0 \cdot 14}(\mathrm{NS}) \\
1 \cdot 25(\mathrm{NS})\end{array}$ \\
\hline
\end{tabular}

NS= not significant. 
the control group agreed with those predicted by Hardy-Weinberg equilibrium. The table gives the relative risks with $95 \%$ confidence intervals 9 associated with the Fok 1 genotypes. We have illustrated the effect of the 2,2 genotype (homozygous absence of the Fok 1 restriction site), contrasted with other genotypes, as this was the genotype that was associated with an increased risk of type I diabetes. There was no increased risk associated with the 2,2 genotype either in the RA group overall or in the 140 DR4 positive patients with RA.

\section{Discussion}

Although it is generally believed that the HLA linked component of susceptibility to RA constitutes a minority of the whole genetic contribution to this disease, it is not clear how many other genes might also be involved. ${ }^{16}$ Indeed, no other susceptibility loci have been identified with certainty in patients with RA. In the nonobese mouse model of autoimmune diabetes there are at least four genetic loci that have been incriminated in susceptibility ${ }^{10} 11$ and in the human disease there is evidence for at least one non-HLA locus. ${ }^{7}$ Our results indicate, however, that this locus in the insulin/insulinlike group factor 2 region on chromosome $11 \mathrm{p}$, though relevant to HLA-DR4 dependent diabetes, is not common to all DR4 related autoimmune diseases, including $\mathrm{RA}$.

K D Pile is in receipt of the Dorothy Eden Fellowship of the Arthritis Foundation of New Zealand. This work was financially supported by a grant from the Arthritis and Rheumatism Council.

1 Wordsworth P, Bell J. Polygenic susceptibility in rheumatoid arthritis. Ann Rheum Dis 1991; 50: 343-6.

Wordsworth B P, Lanchbury I S S, Sakkas L I, Welsh K I Panayi G S, Bell J I. HLA-DR4 subtype frequencies in rheumatoid arthritis indicate that DRB 1 is the major susceptibility locus within the HLA class II region. Proc Nat Acad Sci USA 1989; 86: 10053-9.

3 Wordsworth B P, Bell J I. The immunogenetics of rheumatoid arthritis. Semin Pathol Immunopathol. In press.

4 Lanchbury J S S, Jaeger E E M, Hall M A, et al. Strong primary selection for the Dw4 subtype of DR4 accounts for the HLA-DQw7 association with Felty's syndrome. Hum Immunol 1991; 32: 56-64.

5 Wordsworth B P, Buckley K D, Lanchbury J S S, et al. HLA heterozygosity contributes to susceptibility to rheumatoid arthritis. Am $\mathcal{F}$ Hum Genet. In press.

6 Deighton C M, Walker D J, Griffiths I D, Roberts DF. The contribution of HLA to rheumatoid arthritis. Clin Gene 1989; 36: 178-82.

7 Julier C, Hyer R N, Davies J, et al. Insulin-IGF2 region on chromosome 11p encodes a gene implicated in HLA-DR4 dependent diabetes susceptibility. Nature 1991; 354: 155-9.

8 Wordsworth B P, Allsopp C E M, Young R P, Bell J I HLA-DR typing using DNA amplification by the polymerase chain reaction and sequential hybridisation to sequence-specific oligonucleotide probes. Immunogenetics 1990; 32: 413-8.

9 Kirkwood B R. Essentials of medical statistics. Oxford Blackwell, 1988: 174-6.

10 Todd J A, Aitman T J, Cornall R J, et al. Genetic analysis of autoimmune type 1 diabetes mellitus in mice. Nature 1991 ; 351: 542-7.

11 Cornall R J, Prin J-B, Todd J A, et al. Type 1 diabetes in mice is linked to the interleukin-1 receptor and Lsh/Ity/Bcg genes on chromosome 1. Nature 1991; 353: 262-5. 